\title{
A Survey: Sentimental Analysis on Product Reviews Using (MLT) Machine Learning Techniques and Approaches
}

\author{
Usman Nawaz ${ }^{1}$, Abid Ali ${ }^{2}$, Usman Ahmed Raza ${ }^{3}$ 'Kiran Shehzadi ${ }^{4}$ \\ ${ }^{1}$ Lahore Leads University, Lahore, Pakistan, usmannawaz065@gmail.com \\ ${ }^{2}$ Lahore Leads University, Lahore, Pakistan, royabidali@gmail.com \\ ${ }^{3}$ Lahore Leads University, Lahore, Pakistan, usmanahmedraza@gmail.com \\ ${ }^{4}$ Lahore Leads University, Lahore, Pakistan, Kiranmehar951@gmail.com
}

\begin{abstract}
Social media sites are used today for the development of different types and nature of customers those use such benefits which are often shared by people on social media symbolic or textual opinions, ideas, and feelings. This attitude and orientation draw attention to research and analyze sentiments through online data about customer interest. Therefore, the sentimental analysis idea is proposed. This is among the various uses of Natural Language Processing (NLP) and Machine Learning Analysis (MLA) is very common. The main task of sentimental analysis is the classification of sentiments automatically into three categories that are positive, negative and neutral. Many classification researches are conducted over the years to know the exact feelings and situations of sentimental emotions of people. Classification, fuzzy and clustering, is used. To know the sentiment analysis of the people's accurate feeling and situation, many times over the years classification research was conducted in past. The accuracy of classification is finding more in Fuzzy based. Fuzzy based classification finds more accurate and for comparative study execution Classical Text Classifications Model is used. In comparative performance, this study shows the possibility of implementing the proposed method able to provide more accurate results when it comes in comparison with conventional classifiers. In this article we have discussed different researchers worked on the method of sentiment analysis and classification. This article also shows the importance of extracting comments and analyze sentiments.
\end{abstract}

Key words: Sentimental Analysis, Machine Learning, Supervised Machine Learning, Emotion Detection

\section{INTRODUCTION}

In this advanced period, many people extensively use internet, share comments and recommendations, and personal experience with each product and policy like smoking issues but the proper analysis is difficult to choose according to the suggestions and any context where people's opinions become harsh. Evaluate user's reviews, negative or positive, towards a specific case for purchase or examination [1]. In this situation researchers want to announce a sentimental analysis of this dataset that is for finding the polarity around a particular element. So Professor, the biased computer machine, can be invented to capture these quick decisions in a precise way. The main point is to extract from the file meaningful data and user feedback analysis express sentiments because most people use this method to find satisfactory views of others in search of comments. However, some of the issues raised in prospecting for Opinion Management (OM) such as few words show allegorical meaning differently and effectively. For example, the word "small" gives a negative and if we use "this" it is a positive feeling concerning a particular element like the little smartphone "gives the word a positive look here." The flip side is that we say this is a "Small Battery Timer" negative views would appear in the analysis of sentiments [2]. Categorizing a user's written text is a critical task. This classification is generally considered at many levels [3]: document-level,[4] sentence level[5] and dimension level[6].At the document level, SA intends to prepare a Feeling Name Report communicate a positive or negative result or gratitude. While full SA is considered at the sentence level plan to sort out the sentiments conveyed in each sentence. There is no such difference between document level and level classify at the sentence level because the sentences are only short documents. Document-level and sentence level classification of these important details does not give everyone the sentiment, they need parts of the material needed in many applications, to get these points of interest. So we need the side level intends to evoke sentiments like this certain parts of the elements. The first step of selection aspects and institutions because there are different opinions about it similar entities to perform classification tasks for SA, machine learning techniques and dictionary-based strategies. In some cases, the combined method, vocabulary base and machine learning methods are employed [7], [8]. Also, it depends on the dictionary-based strategies. The sentiment-based analysis approach consists of two sub-section categories, I-e dictionary database and group database. They tackle the challenges of big data and machine learning methods based on sentiment analysis. These learning methods are not supervised but semi-supervised learning approaches/methods. Supervised learning is based on available training documents where positive results appear. 
Vector Machine Support (SVM), Naïve Bayes (NB), Decision Tree (DT), Max Entropy (ME), and Neural Network (NN) are very useful.NB-supervised learning algorithms are the most common Classification was used to analyze sentiments $[9,10]$.

Unsupervised learning is useful for solving problems tagging unlabeled data because it is easier. It affirms and implements the superior result used by many researchers [11, 12].Semi-monitoring techniques are difficult to compare under supervision [13] of the idea of similarity is half that it contains knowledge about the seasons but contains information corresponding to the continuous distribution of classification features [14] if the named data is finite, execution is halved modified method to restore external performance for comparison of other technologies. In recent years, various technologies have been developed about sentimental analysis and the main tasks in sentimental subjectivity analysis, dictionary law, free extraction, spam.

Expression of opinion and inspection score [15] the main purpose of this study that is to discover the basic abstraction. In the processing and execution of natural language comparative observation in sentimental analysis is used. They announced the different techniques and approaches of this ritual of which strategy is better according to which approach or technique. In fig. 1 different sentiment analysis techniques are applied.

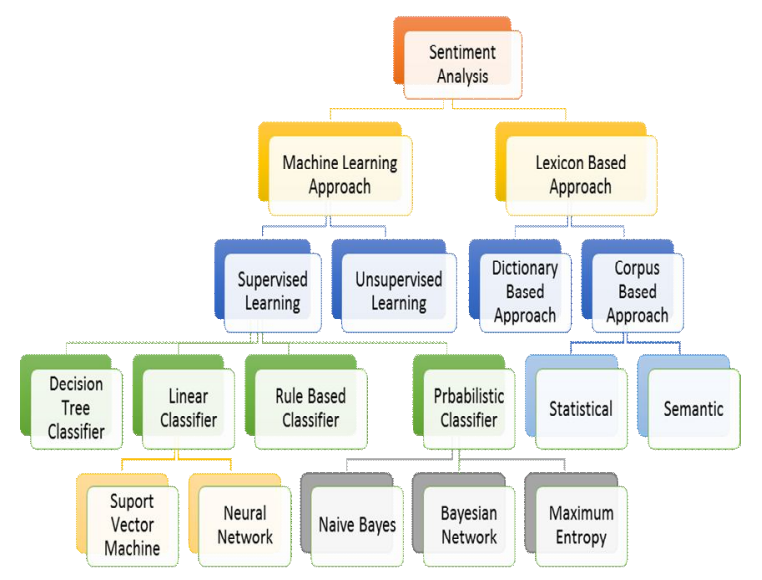

(Fig.1)

In fig. 1 various sentiment analysis techniques.

\section{RELATED WORK}

In this article [16], the author describes a sentimental study analysis using a microblogging based sentimental analysis, deep learning. The main purpose of microblog sentiments analysis is to find out user behavior and opinions about hot events in this proposed method. The author description of the new technical solution in this solution, a crawler has crawled some data from a microblog. Distance due to the pretreatment of the body, the neural network is bypassed take as an example of inputs and then two sentimental sentences the classification is based on SVM and RNN. The door the last step is to fine-tune the sentimental analysis. Practice this proves that the proposed method is practical for further reinforcement performance compared to other technologies. In [17] this review article, the author discusses publishing data is in different operating systems that are managed by them today use machine learning techniques and vocabulary to do a sentiment analysis and focus specifically on the four direct basic tasks. In the previous post, many used Approaches and classes by comparing their results and accuracy but the most basic SVM and NB in this survey because this approximation is high and has been quoted for new machine learning techniques. Finally, the author has some of them stimuli in other languages are due to its language complexity and need to improve the skills of other languages machine learning techniques.

In this article, the author [18] demonstrates the polarity to analyze sentiments on scattered data using SVM. The door recently, many features, classifications, and algorithms it is suggested but there is no suitable mechanism for each case. However, the author discusses the difference between chi-square between Positive and Negative Categories (CDPNC), a combined algorithm of $\mathrm{DF}$ and $\mathrm{CH}$, to create experimental superiority of classification compared to B. I chose the feature and got a good result the author concludes the analysis that the proposed strategy is innovative good for sentence and document level but needs improvement sentiments at the word level.

In this article [19] the author emphasizes the use of Twitter and the most famous microblogging step to review sentiment analysis. The author pretends to collect a group to examine sentiments and opinion polls objectives to do this, he used various techniques and tools such as Tree See marker selector for POS tagging and differences in distribution between positive, negative, and neutral groups. Empirical evaluations prove our proposal procedures are fruitful and work better than anything compared to the previously proposed methods. The proposed method [20] described many Koreans newspapers are biased because of their biased reporting. The authors of the daily articles intend to state their purpose. Indirectly, they choose a technique for forgetting some important thing she uses facts or occasionally one-sided terms to reinforce them, evaluating them and it is difficult to evaluate the nature of their writings. In this article, we propose a strategy to identify political bias in the country Korean articles by making first word vectors and do sentence vectors, and in the second step do the DBN tutorial with them take for calculation using SVM and finally use the return path Bias, orientation. We used our recorded data set political bias has recently led to a recurrence.

After reading this article [21], sentimental analysis of the text Content is a vital part of knowing how to manage a person they understand the content of the text and have an extensive program in the file construction. We learned with deep interest and success the suggestion is to work on sentiment analysis through deep learning models. In this article, we propose a framework calledWord2vec + Convolution Neural 
Network (CNN). We are the first use the word 2vec suggested by Google to record the vector display the words, which will be the input to tin is motivated to use the word $2 \mathrm{vec}$ choice even display word and place separate words. We also use Parametric Correction Linear Unit (PReLU), normalization and leakage technology to improve the accuracy and generalization of our model. Through this test, we achieved 45.4\% accuracy in this matter dataset, which performs better than other components of neural components that show system as recursive neural sequence (RNN), recurrent matrix neural network (MV-RNN).

In this research [22] over the past decade on the internet, not only did people gradually use it customers, however, are more like measures for online web data. Topics in these forums were related to health and treat fertility problems and treat them in the lab fertilization. This article specifically introduces machine learning examines these ranking hypotheses in the discussion literature. Current research has few goals. Original the aim was to address different interpretations of the functions. The second was to think of several dictionaries of sentiments and select a specific location for this particular article on our results to reach an average of $\mathrm{F}$ of 0.805 .

The article [23] examined the opinion on the Romanian Urdu language because the work is researched in languages other than English very little, so the author loved the Romans Camp using Waikato tags Knowledge Analysis (WEKA).The author uses three examples with the Romanian performance of Urdu Algorithms (KNN, Naes Bayesian, Decision Tree). Distance the application and comparison of these algorithms analyzed naivety the Bayes algorithm performed a more precise task accordingly for other algorithms.

Reference descriptive [24] sentimental analysis using domain Adaptation algorithm for semi-supervised bias an approach to increase its performance. In this approach, views are classified using maximum entropy (ME) and allocation then we group the weight of the classified words according to the point Wise Mutual Information (PMI). Domain compatibility the most important complication in the sentimental analysis is the author this is stated in his proposed method relatively good result compared to the base method.

In this article [25], the author examines different strategies and other approaches of researchers and their comparison these measures are introduced by the new concept of May ranking for quality. The author suggested a vague concept with a useful new approach to Basic text strategy. It shows high performance compared to traditional techniques

In this article [26], the author of semicircles demonstrates a dictionary-based approach to analyzing Twitter sentiments. Due to the function of the ordinary dictionary based on the unique dictionary methods provided before constant and consistent sentiments, the polarization of words does not pay much attention to their uniqueness. Senti circles captures the joint event examples of words in different settings in the tweets to catch numerology and quality improvement. Quality maximum in the dictionary of sentiments if necessary. The writer suggests a method that considers cognition inference at item level and Tweet level. For this in this article [27], the author has used this proposed method in three datasets on Twitter using three distinct sentiments dictionaries to determine the previous sentiments of the word. Calendar this proposed approach is fundamentally superior baseline in accuracy and F-scale at the entity level. The mentality of the proposed approach to sentiments at the Tweet level define superior performance. However, a force is transmitted with an accuracy of $4-5 \%$ in two data sets in the third data set it lags barely $1 \%$ on the F scale.

In this article [28], the author explains the growing interest due to the expansion and development of social communities and networks of experts, researchers, and decision-makers he faces the challenges of online polling on the ground comments like this include unstructured settings with informal languages and terms and mixed languages can be imagined. Many challenges due to the complexity of the Arabic language and number of research publications and predefined data sets why I collected and analyzed. Basically, two Managed (group-based) methods without supervision (According to the dictionary) decide on the most intense surveys. The writer used the second method and it was a huge glossary of sentiments and tools based on SA Dictionary Etc. The proposed method showed unusual results. In terms of predictive accuracy, compared to a keyword-based approach. People who use the internet to make their assumptions on various topics up to this article it depicts a framework for examining sentiments on Twitter Sorts tweets according to their year, positive or negative Party level tweets [29]. Managed to learn workbooks, For example, we often type incorrectly for tweets "but, and conditional, for example," if ", because of their unusual semantic traits. These workbooks likewise, the degree of selection is close to the selection limit. Extensive number of Tweets you recommend you are not sure that you are completely out of this business Tweets .To address these two issues, this article suggests a framework that improves organ-managed learning characterization using semantic and sensory principles computational resources of the method suggested by the author. The assessment was conducted on two Twitter companies that are openly available to identify them sufficiency [30].

In this article, the author has used Twitter the most famous stage of microblogging for review sentiment analysis [31]. The author explains naturally collect a booklet to explore sentiments and draw out comments objectives for this author use different techniques and tools like Tree Tagger for POS tagging and notes distribution for difference between positive and negative and Neutral groups Experimental evaluations prove that we have Proposed actions are fruitful and performance is superior to perform anything compared to the previously proposed methods.

This article deals in detail with the issue of human perception sentiments caused by network images on the internet on a scale, to consider both visuals and logical propositions, for example, notes on photos, inscriptions, etc. The challenge lies in the "semantic gap" between low-level images as well as larger image views. Also, no comments/tags in the majority of 
network images on the internet show another part New Challenge Unsupervised Sentiment Analysis (USEA), a system for social media images on the internet has been proposed to solve these two problems. This approach takes advantage of relationships between visual material and relevant data to relate "semantic gap" in image predictions sentiments through experimentation with two large datasets, show that the proposed method is strong two problems [32]. Recently, social media has become an emerging wonder due to the extraordinary and fast progress of information technology. People use social networks on the internet communicate daily to discuss their evaluation with each other around a wide range of themes, elements, and sections. There are too many clients or auditors' sentiments that are created and shared every day. Twitter has featured this network is one of the most widely used small networks on the Internet Blogging sites extract customer assumptions from the internet network data is not directly forwarded. Perhaps obtained in various ways. The author uses the open-source approach, although Twitter microblog data with free use, the assembly is pre-prepared, dissected, and visualized source devices to perform sentiment analysis and hypothesis screening to review online audits involving the client especially the two giant British retailers, Tesco Asda stores in Christmas 2014. Customer gathers using sentiments can be costly and time consuming traditional techniques, for example, polls. Sentiments analyzing customer feedback makes the job easier for organizations to understand their centralized appreciation change the business segment and the perception that their customers see about their components and departments, which they additionally offer knowledge of future progress and basic actions leadership arrangements [33].

The inevitable contradiction between traditional AI frameworks And human knowledge is the human capacity for real processing information gathered from the lifespan of learning experience making informed choices. It allows people to easily adapt to the new conditions that artificial intelligence is coming due to the lack of special standard positions and speculation capabilities, the catastrophe is short-lived. Common sense learning also gives information to which institution enables these people to act effectively in social situations where this learning is usually expected. Logic contains information that people use at the very least, collecting them is very annoying oath - commitment. In this work, the author has used a combination of hierarchical clustering and dimensional reduction to naturally find the basics for noun and verb ideas in SenticNet [34]. This circular handle is allowed in general, it expands the scope of logical knowledge deployed previous bugs SenticNet was all about collecting this some kind of learning to analyze sentiments anyway due to the failure of the SenticNet 4 summary, it was severely tied. It overcomes these limitations by using concepts thus the first is created by the hierarchical method cluster and dimensional reduction.. This article is presented a new technique for predicting sentiments and grouping make progress by reviewing code review comments [35]. Edit code from the source control store to remove the recovery move from three years ago to two years open source projects were symbolic and items were extracted to identify sentiments, For example, calm, annoyance, and awe. Feeling the strongest comments of the code review have been measured so far Neutral vs. rudeness. He presented the strategy used $67 \%$ accuracy in recognizing oppression comments.

This article illustrates the strategy of transient discrimination links between news articles using phrase insertion investigation. Newsreader words were coded using unusual character and separating space word codes are categorized by type, for example, name, verbs, prepositions, and adverbs. Space for each word and separation of the verb on the left, right, past, and next to this sentence is registered as an element. Links between different sentences using nouns as anchors and verbs were verified as a proven connection occasion was measured and the accompanying design was presented as an option the tree of this model was implemented experimentally using time questions the event sequence tools. The result of the proposed system $67 \%$ showed accuracy according to the correct event sequence and the improvement in the feeling of a news-based ranking as well .There was an opportunity to see if the weight schematic diagram was tested communication can be according to the situation expression strategy used to analyze feelings of fear and contentment An arrangement that shows the sharp edges of a special occasion and the type of their temporal relationship [36].

Predicting stock prices is essential in regulating business activities. The purpose of this article is to collect using a model to predict stock price evolution sentiments from the network through the internet. It is not like the past at all methods where there are general mindsets or evaluations look, have feelings from some places internal organization to view inventory forecast. Related topics and sentiments naturally separate texts are loaded using a suggestion in the message. This strategy is available in addition to thematic models. Furthermore, this article describes an adequacy assessment analyze sentiments in predicting your committed stocks it means large-scale experimentation. Due to the average accuracy of more than 18 shares in a one-year deal, the author proposed technology $2.07 \%$ better performance than only model using historical costs compare strategies only for difficult stocks predict, this strategy has reached a better accuracy of $9.83 \%$. From the historical cost method, it is $3.03 \%$ better than the human's comment strategy [37].

This study focuses on the analysis of sentiments at the lateral level. Where the goal is to discover and collect sentiments institutions mentioned in the documents or their aspects. Summit - conference to depict the current state of the art, which shows the tremendous progress that has been made so far in finding the goal, which can therefore be an element or part of it, and the sentiments associated with it. At the height level, sentimental analysis leads to extremely subtle sentiments data that can be useful for applications in different locations. Current arrangements are ranked based on their offer or non-submission a strategy for discovering aspects, analyzing sentiments, or both. Besides, a failure in terms of the type of algorithm used for each study discussed 
implementation merged. For a little encouragement, evaluate the various techniques suggested, call to institutionalize the evaluation strategy which involves the use of shared datasets. Concept-based evaluation in this exam is considered as one of the exams discussed and separated the most encouraging future research course [38].

Sentiment Analysis (SA) or Survey (OM) for them since then, it has recently become the focus of many analysts. Online content screening is both useful and desirable statistical studies and logical studies of psychology and social perspectives, political polls, business insights, web-based shopping framework and so on. These days if you need to buy one, the first consumer item to the customer review and view in free web discussions about the item later commented quickly signs of this talk on the internet talking about new and a measurable data source with many applications. This method is for recognizing and separating mental data raw information is known as sentiment analysis. The author discusses various sentiment classification techniquesfFeel the classification tools. This article has also featured a study of the evaluation or survey test [39].

\section{1 initiative Procedure of Sentimental Analysis}

Many use the internet for their sentimental analysis of opinions and extraction from similar fields are research various NLP-derived techniques are used, structured and extracting unstructured data and information sentimental recall is a multilingual problem, not one [40].

Data is the most common step. Buy data and data pretreatment is essential in the sentimental realm, an analysis of the concept of sentiments is provided by Nosokawa and Yi in 2003 and the concept of polls by Dave, Lawrence in 2003. In [41, 42, 43] this study was conducted about polarity through machine learning [44] based on dictionary and synthesis [45]. In [46] the author on specific problems in SA and So OM suggested ranking levels (document Level, sentence level, aspect level, adaptive sentiments Analytical, lexical) specified for different heights Challenges and issues.

\subsection{Sentiment Analysis Levels \\ 2.2.1 Document Level}

The whole report is executed and then select document positive or negative assumption. The primary data unit is a single archive of stubborn content. In this description of the document level, an individual review is about a single point. Be like that you may do the same, at the expense of online topics or magazines sentences may appear. Customers can compare an item and another that has comparative and therefore standard features research in web gatherings and magazines is not tempting. There are two basic ways to work with document levels sentiment test: circuit learning and unattended training. The circuit approach predicts these classes have a limited order an archive should be compiled over preparation information is available for each category. The least problem status at the point where there are two classes: positive is also negative. Can drive expansions? It also includes a class that is neutral or has little or no discrete digital criteria on which the archive should be set (e.g. Five-star frame used by Amazon). By preparation information, the frame is done in assembly a model using one of the regular collection accounts, for example, SVM, Naive Bayes, Strategic Regression, or KNN this grouping is used to mark new records in files different ranking categories. At a point that is a number, the estimate (within a limited range) must be assigned to the record relapse can then be used to predict stimuli prepared for the report (for example, on Amazon five stars Frame positioning). A look at this issue has shown both are extremely accurate and durable the report is said to be a straightforward package of words [47]. There are also two main methods to choose from phrases: a range of predefined POS styles can be used to select these phrases or words of sentiment can be words and phrases it can be used [48]. This closes the comment level the pool has its points of interest. The disadvantage of this position is that it has the advantage of being public maximum content of sentiments about a particular substance from a record. Damage is a sentiment that characterizes him different items cannot be removed from one item independent [49].

\subsubsection{Sentence Level}

An individual case may even involve several assumptions similar when we need more good perspective of characteristic assumptions in the document about the elements that we should have go to the sentence level. At the level of the skewed sentence research, the party (positive/negative/non-partisan) of each sentence is confirmed. Similar report level descriptive strategies can be linked to sentences level layout issue. Objective and subjective sentences must be discovered to contain mental sentences rank the words that help determine the orientation around the level. The limb is then described into positive and negative categories. The slope of the sentence surface if you stumble upon this mind, accumulation is not tempting sentences, having repetition is useful. A direct sentence is used in most strategies and a modified approach to dealing with sentence order two categories [50]. The boot was systematically recommended by Hay with the ultimate goal reduce the scale of physical work required during startup large group setting $[50,51]$. Abnormal this method has been proposed due to the substantial reduction in Bang W. I [52]. This is the main hypothesis of their approach adjacent sentences should have the same mentality specify. As previously defined, this is an approach assessment test at sentence level or in light of guided learning or unsupervised learning [53]. The final method that is inherently comparable to the Turney method, with the difference that it uses the possibility of recording a variable instead of the PMI and the number of words that the seed gives words are used to discover SO in a sentence much larger [54].

\subsubsection{Aspect Level}

The previous two methods are admirable for each of them the whole story once again refers to every strange sentence to an isolated element. Aspect-based sentiment analysis (also 
sentiment-based analysis is exploring a case that highlights the recognition of all sentiments phrases in a particular report and their angles. The hint is great for grouping the display level assumption dealing with a more specific way of dealing with final extraction. Phrases containing hypothetical words are identified description of expression level completed. It can be useful or undesirable. Classic approach, used by many business organizations, clear evidence from all perspectives on a set of elements, polls are at the center of the phrase (NP) and beyond only keep NPs whose repetition is temporarily over threshold set [54]. It is a method of reduction turbulence in NP is diagnosed from time to time, right? Feelings about a substance can be effectively removed [55]. In different situations, be like this if necessary the parties are also important, it may not be the result quite accurately another way to approach a point of view. The best-known clue is to use a dependency analyzer uses popular ranking phrases for more information angles (actually even transverse angles) [56]. In the same way, we can view the ID issue from a point of view as a data mining problem then use the group to make a replacement, for example, a random work context (CRF) based on workbook discover aspects [57]. Speech cancellation can occur locally in situations like this, this level of sentimental analysis is necessary enough. By the way, if there are phrase sentences, words separate from hypothetical words, nationwide research is not desirable. Words that have a sound they are believed to be exceptionally close to each other Idiom. This is the last point in every respect they are controlled by a weight criterion for the poles of each idea phrases are inverted by separating evaluation of perspective and expression [58].

\subsection{Sentiment Analysis Techniques}

There are two main ways to analyze sentiments machine learning approach and the dictionary-based approach. The next is the hybrid approach. This approach a combination of machine learning and dictionary-based technique. The machine learning method is used to do the job the task of sentimental analysis in the famous machine learning textual data approach. This can be a machine learning approach this is mostly divided into supervised and unsupervised ranking relies on a glossary-based approach dictionary. The dictionary-based method is divided into subcategories vocabulary and group-based tasks $[59,60,61]$.

\subsubsection{Lexicon Based Approach}

In these unsupervised techniques, classification is performed it takes into account the polarity-based characteristics of people's views it then analyzes their positive or positive opinions negatively, this glossary is called sentiment. To compile the file the word net sentiment is used manually but it is difficult and this process is time-consuming and does nothing alone. It needs two more automated approaches to do these Sentiments automatically. These two approaches are automated by the following knowledge [59, 60, 61].

\subsubsection{Dictionary Based Approach}

This introduces the basic policy for dictionary-based technologies [62], [63] first, he determines the polarity of a word after finding it in the dictionary, its synonyms, contradictions, and popularity. As a WorldNet [64], [65], [66], or word finder group [67] Tracks duplication after finding a new word then add it to your keywords. Repeat this process until Do not look for new words, then the manual search continues to correct the error. In [68] this author has used this method for 30 attributes that contain limitations so note that comment words cannot be searched. In this approach, there is a basic penalty that is not penalized comment words in a specific context $[69,70]$. The next author has it the rule-based approach that was presented in most cases was a good performance in the letter.

\subsubsection{Corpus-Based Approach}

The stack-based method performs relatively well with accuracy solve the problem of finding specific comment words special trends are a big feature that Corpus trusts an approach that does not have a dictionary-based approach. The door [71] the author used the Conditional Randomness Fields (CRF) method to extract comments through manual learning techniques. This is also by author [72] to determine the compatibility of a multidisciplinary polar pattern Algorithm used in Chinese language studies. They work on different elements and get good results. The author [73] has used two levels of the CRF model for relative generation relationships without interdependence. Shows the result as it is compared to other models, it is relatively good and proven an effective tool for individual use of the blog-based approach it is not significant compared to the dictionary-based method because it cannot handle a large group, but it is particularly useful speech and opinion this method is used through a statistical or semantic approach.

\subsubsection{Statistical Approach}

Used for research on concurrency patterns and opinion extraction words in this way. It is used for many related matters as a program for analyzing sentiments. To find polarity a large group of annotated words [74, 75].

\subsubsection{Semantic Approach}

To demonstrate it, it relies on this principle to analyze similar accounts its semantic value is straightforward. It is used differently applications to create a basic lexical form for expression sentiments use nouns, verbs, adjectives, and adverbs the analysis used by the author in [76, 77]. It has been used in this author both methods create shortcomings that people have online review the analysis of sentiments and the use of features and basics based on the sentence. They offer high performance in research weakness $[78,79,80]$.

\subsubsection{Hybrid Approach}

A combination of machine learning and dictionary-based is called the hybrid approach. Some research analysis techniques that improve the hybrid method performance in 
ranking sentiments. In this author, he used these two techniques together on a sentimental level high-resolution analysis and performance [77].

\section{PROPOSED METHOD}

In this technique ensuing preprocessing job has finished categorizing sentiment analysis as of reviews.

\subsection{Gathering of Dataset}

The dataset is composed as of Amazon in js arrangement. Every js folder holds the number of reviews. Dataset has reviews on Cameras, Laptops, Tablets, Cell phones, TVs, video surveillance.

\subsection{Preprocessing}

In this method tokenize, break chat deletion, stopping, punctuation marks deletion, et cetera, that completed. It has transformed into a container of arguments. Preprocessing is essential in sentiment examination and view removal.

\subsection{Mark Generation}

In mark generation, each stretch has examined and planned sentimentality mark. To compute sentimentality mark dataset that matched with view lexicons i.e. 2007 positive arguments and 4784 negative arguments and designed sentimentality mark for each judgment.

\subsection{Sentiment Classification}

By mark and dissimilar structures dissimilar machine learning procedures useful and dissimilar correctness dimensions planned. The technique uses the next jobs to categorize the sentiment examination by machine learning methods.

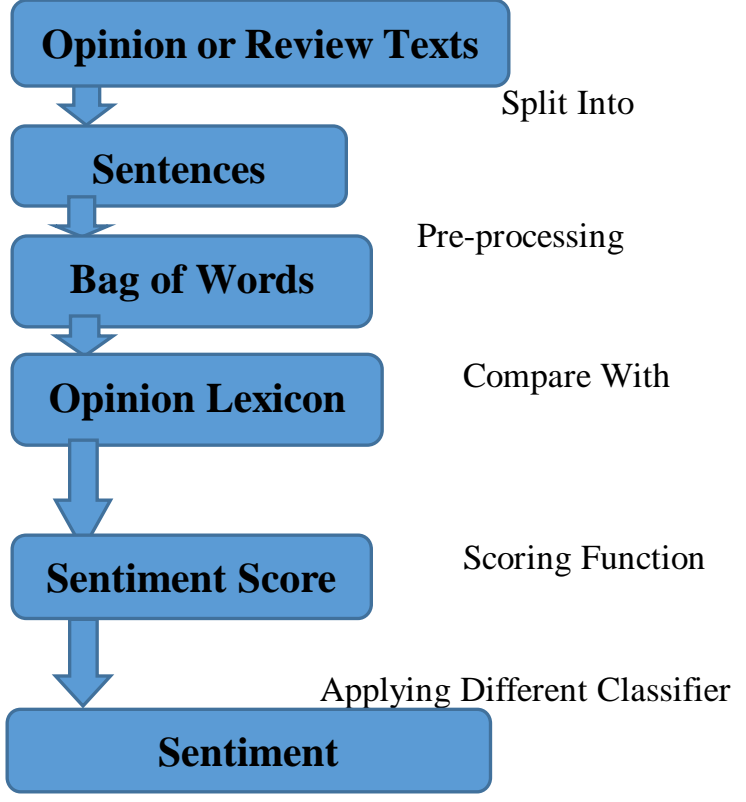

Fig. 2 Suggested methodology of sentiment analysis.

\section{EXPERIMENTAL RESULTS}

\subsection{Dataset Account}

The contains reviews of Laptops, Cameras, Tablets, Cell phones, TVs, video surveillance composed from Amazon. That is in the procedure of json folders and every json folder comprises the number of analyses (Fig.3); Table 1).

\subsection{Classification Outcomes}

See (Fig. 4)

Table 1 Dataset and reviews calculation.

\begin{tabular}{|l|l|}
\hline Dataset name & Number of Reviews \\
\hline Camera & 3106 \\
\hline Laptops & 1946 \\
\hline Cell phones & 1918 \\
\hline Tablets & 1894 \\
\hline TVs & 1596 \\
\hline Video surveillance & 2597 \\
\hline
\end{tabular}

Fig. 3 Graphical representation of the number of reviews

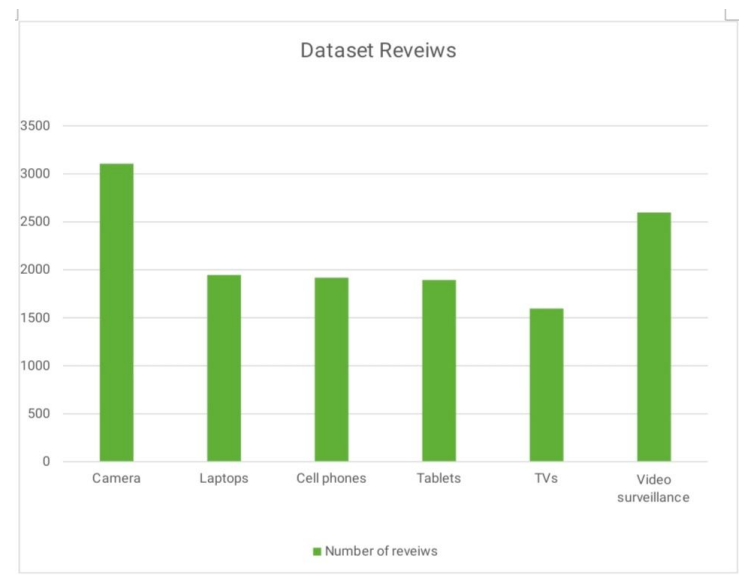

Table (2) Calculation parameters for classifiers of datasets

\begin{tabular}{|c|l|l|c|l|l|c|}
\hline \multirow{2}{*}{ Dataset name } & \multicolumn{5}{|c|}{ Classifiers } \\
\cline { 2 - 7 } & \multicolumn{2}{|l|}{ Naïve Bayes } & \multicolumn{2}{l|}{ SVM } \\
\cline { 2 - 7 } & Accuracy & Precision & $\begin{array}{c}\text { F } \\
\text { score }\end{array}$ & Accuracy & Precision & $\begin{array}{c}\text { F } \\
\text { score }\end{array}$ \\
\hline Camera & 98.17 & 98.30 & 99.03 & 93.54 & 93.58 & 96.66 \\
\hline Laptops & 90.22 & 90.01 & 94.74 & 88.16 & 88.52 & 93.71 \\
\hline Cell phones & 92.85 & 91.64 & 95.64 & 92.85 & 91.64 & 95.64 \\
\hline Tablets & 97.17 & 98.73 & 98.31 & 84.12 & 84.31 & 91.37 \\
\hline TVs & 90.16 & 90.17 & 94.72 & 88.49 & 85.56 & 93.89 \\
\hline Video surveillance & 91.13 & 89.95 & 94.71 & 79.43 & 84.25 & 88.53 \\
\hline
\end{tabular}

Fig. 4 Graphical representation of the experimental outcomes. 


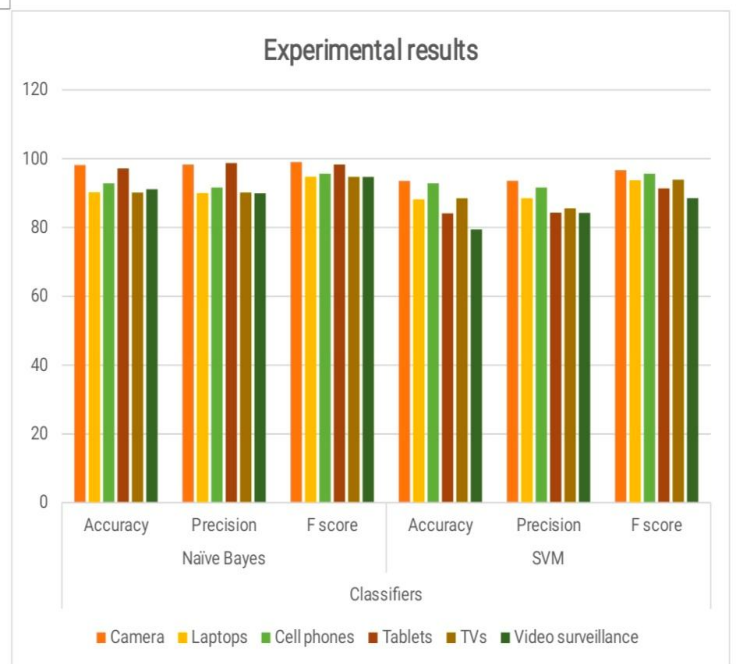

5 CONCLUSION

As the use of the internet has expanded, we have announced in this survey the importance of polls and sentimental analysis is the development of an area as individuals share their views, opinions, and views on the web. People share their views and opinions in various fields. Social media is creating enormous of data and that is essential to analyze and discover perceptions as that data. This report products reviews dataset takes as of Amazon webs having 6 product reviews of Camera, Laptops, Cell phones, Tablets, TVs, and Video Shadowing. There is a planned procedure that depends on the dictionary method beneath the lexicon-based method that is cast-off with machine learning techniques. Sentiment analysis that completed on each object analyses and then categorized using machine learning procedures, for example, NB and SVM. Table number 2 displays the correct dimensions for Naive Byes and Support Vector Machine analyzer aimed at the dataset. Naive Bayes analyzer acquired 98.17\% accuracy for Camera reviews and SVM acquired 93.54\% accuracy for Camera reviews. For upcoming efforts, on a similar dataset, feature level sentiment analysis can increase outcomes and as of that technique, I can acquire what accurately people enjoyed or not enjoyed. Like, Camera's feature, image size, assembly, lens, image value, et cetera. Feature level is the best method and provides better outcomes in sentiment analysis.

\section{REFERENCES}

[1] I. Kaur and G. Lal, "Sentiment Analysis of Amazon Canon Camera Review using Hybrid Method," International Journal of Computer Applications, vol. 182, no. 5, pp. 25-28, 2018, doi: 10.5120/ijca2018917545.

[2] A. P. Jain and P. Dandannavar, "Application of Machine Learning Techniques to Mineral Recognition," Computer, no. October, pp. 628-632, 2016, [Online]. Available: http://innovexpo.itee.uq.edu.au/2001/projects/s3693 57/thesis.pdf.

[3] A. Kumar and R. Rani, "Sentiment analysis using neural network," Proceedings on 2016 2nd International Conference on Next Generation Computing Technologies, NGCT 2016, no. October, pp. 262-267, 2017, doi: 10.1109/NGCT.2016.7877425.

[4] G. Preethi, P. V. Krishna, M. S. Obaidat, V. Saritha, and S. Yenduri, "Application of Deep Learning to Sentiment Analysis for recommender system on cloud," IEEE CITS 2017 - 2017 International Conference on Computer, Information and Telecommunication Systems, pp. 93-97, 2017, doi: 10.1109/CITS.2017.8035341.

[5] M. S. Neethu and R. Rajasree, "Sentiment analysis in twitter using machine learning techniques," 2013 4th International Conference on Computing, Communications and Networking Technologies, ICCCNT 2013, 2013, doi: 10.1109/ICCCNT.2013.6726818.

[6] M. Y. Day and Y. Da Lin, "Deep learning for sentiment analysis on google play consumer review," Proceedings - 2017 IEEE International Conference on Information Reuse and Integration, IRI 2017, vol. 2017-Janua, pp. 382-388, 2017, doi: 10.1109/IRI.2017.79.

[7] P. Yang and Y. Chen, "A survey on sentiment analysis by using machine learning methods," Proceedings of the 2017 IEEE 2nd Information Technology, Networking, Electronic and Automation Control Conference, ITNEC 2017, vol. 2018-Janua, pp. 117-121, 2018, doi: 10.1109/ITNEC.2017.8284920.

[8] A. K. Uysal and Y. L. Murphey, "Sentiment Classification: Feature Selection Based Approaches Versus Deep Learning," IEEE CIT 2017 - 17th IEEE International Conference on Computer and Information Technology, pp. 23-30, 2017, doi: 10.1109/CIT.2017.53.

[9] A. S. Zharmagambetov and A. A. Pak, "Sentiment analysis of a document using deep learning approach and decision trees," Proceedings of the 2015 12th International Conference on Electronics Computer and Computation, ICECCO 2015, 2016, doi: 10.1109/ICECCO.2015.7416902.

[10] R. Ghosh, K. Ravi, and V. Ravi, "A novel deep learning architecture for sentiment classification," 2016 3rd International Conference on Recent Advances in Information Technology, RAIT 2016, pp. 511-516, 2016, doi: 10.1109/RAIT.2016.7507953.

[11] G. Gautam and D. Yadav, "Sentiment analysis of twitter data using machine learning approaches and semantic analysis," 2014 7th International Conference on Contemporary Computing, IC3 2014, pp. 437-442, 2014, doi: 10.1109/IC3.2014.6897213.

[12] Z. Hu, J. Hu, W. Ding, and X. Zheng, "Review Sentiment Analysis Based on Deep Learning," 
Proceedings - 12th IEEE International Conference on E-Business Engineering, ICEBE 2015, pp. 87-94, 2015, doi: 10.1109/ICEBE.2015.24.

[13] A. Hassan and A. Mahmood, "Convolutional Recurrent Deep Learning Model for Sentence Classification," IEEE Access, vol. 6, no. c, pp. 13949-13957, 2018, doi: 10.1109/ACCESS.2018.2814818.

[14] S. Umar, M. M, F. Azhar, S. Malik, and G. Samdani, "Sentiment Analysis Approaches and Applications: A Survey," International Journal of Computer Applications, vol. 181, no. 1, pp. 1-9, Jul. 2018, doi: 10.5120/ijca2018916630.

[15] B. Liu and L. Zhang, "A survey of opinion mining and sentiment analysis," Mining Text Data, vol. 9781461432, no. 3, pp. 415-463, 2012, doi: 10.1007/978-1-4614-3223-4_13.

[16] L. Vinet and A. Zhedanov, "A 'missing' family of classical orthogonal polynomials," Journal of Physics A: Mathematical and Theoretical, vol. 44, no. 8, pp. 1-14, 2011, doi: 10.1088/1751-8113/44/8/085201.

[17] R. Aggarwal and L. Gupta, "A Hybrid Approach for Sentiment Analysis using Classification Algorithm," International Journal of Computer Science and Mobile Computing, vol. 6, no. 6, pp. 149-157, 2017.

[18] X. Hu, J. Tang, H. Gao, and H. Liu, "Unsupervised sentiment analysis with emotional signals," $W W W$ 2013 - Proceedings of the 22nd International Conference on World Wide Web, pp. 607-617, 2013, doi: $10.1145 / 2488388.2488442$.

[19] M. Bhagat, "Sentiment Analysis using an ensemble of Feature Selection Algorithms," 2018.

[20] J. Singh, G. Singh, and R. Singh, "Optimization of sentiment analysis using machine learning classifiers," Human-centric Computing and Information Sciences, vol. 7, no. 1, 2017, doi: 10.1186/s13673-017-0116-3.

[21] S. Wu, Y. Liu, J. Wang, and Q. Li, "Sentiment analysis method based on kmeans and online transfer learning," Computers, Materials and Continua, vol. 60, no. 3, pp. 1207-1222, 2019, doi: 10.32604/cmc.2019.05835.

[22] Bahwari, "Sentiment Analysis Using Random Forest Algorithm-," Journal Of Information Technology AND ITS UTILIZATION, vol. 2, no. 2, pp. 29-33, 2019, [Online]. Available: https://www.researchgate.net/publication/338548518 _SENTIMENT_ANALYSIS_USING_RANDOM_F OREST_ALGORITHM_ONLINE_SOCIAL_MEDI A_BASED.

[23] A. I. Baqapuri, "Twitter Sentiment Analysis A Project report submitted in fulfilment of the requirement for the degree of Bachelors in Electrical (Electronics) Engineering."

[24] A. Mandal and M. K. Nigam, "E-R E-R E-R E-R," vol. 1, no. 10, pp. 46-54, 2018.

[25] K. Korovkinas and G. Garšva, "Selection of intelligent algorithms for sentiment classification method creation," CEUR Workshop Proceedings, vol. 2145, pp. 152-157, 2018.

[26] D. Jurafsky, "Sentiment Analysis What is Sentiment Analysis?," Stanford, pp. 1-81, 2017, [Online]. Available:

https://web.stanford.edu/class/cs124/lec/sentiment20 17.pdf.

[27] Q. T. Ain et al., "• Radiotherapy is the gold standard in treating bone malignancy . Effective in 50-90\% expectancy months ).," vol. 8, no. 6, 2017.

[28] Y. Lin, X. Wang, and A. Zhou, "Opinion spam detection," Opinion Analysis for Online Reviews, no. May, pp. 79-94, 2016, doi: 10.1142/9789813100459_0007.

[29] H. Raza, M. Faizan, A. Hamza, A. Mushtaq, and N. Akhtar, "Scientific text sentiment analysis using machine learning techniques," International Journal of Advanced Computer Science and Applications, vol. 10, no. 12, pp. 157-165, 2019, doi: 10.14569/ijacsa.2019.0101222.

[30] P. P. Balage Filho, L. V. Avanço, M. das G. V. Nunes, and T. A. S. Pardo, "NILC_USP: An Improved Hybrid System for Sentiment Analysis in Twitter Messages," Proceedings of the 8th International Workshop on Semantic Evaluation, no. SemEval, pp. 428-432, 2014, [Online]. Available: http://www.aclweb.org/anthology/S14-2074.

[31] U. Swati, C. Pranali, and S. Pragati, "Sentiment Analysis of News Articles Using Machine Learning Approach," International Journal of Advances in Electronics and Computer Science, no. 2, pp. 2393-2835, 2015.

[32] M. M and S. Mehla, "Sentiment Analysis of Movie Reviews using Machine Learning Classifiers," International Journal of Computer Applications, vol. 182, no. 50, pp. 25-28, 2019, doi: 10.5120/ijca2019918756.

[33] V. Batanović, B. Nikolić, and M. Milosavljević, "Reliable baselines for sentiment analysis in resource-limited languages: The Serbian movie review dataset," Proceedings of the 10th International Conference on Language Resources and Evaluation, LREC 2016, no. 2008, pp. 2688-2696, 2016.

[34] J. Rogers, "Presbyterian guidelines for biblical interpretation: Their origin and application to homosexuality," Biblical Theology Bulletin, vol. 37, no. 4, pp. 174-183, 2007, doi: 10.1177/01461079070370040501.

[35] J. Khairnar and M. Kinikar, "Machine Learning Algorithms for Opinion Mining and Sentiment Classification," International Journal of Scientific and Research Publications, vol. 3, no. 6, pp. 1-6, 2013, [Online]. Available: www.ijsrp.org.

[36] A. Hasan, S. Moin, A. Karim, and S. Shamshirband, "Machine Learning-Based Sentiment Analysis for 
Twitter Accounts," Mathematical and Computational Applications, vol. 23, no. 1, p. 11, 2018, doi: 10.3390/mca23010011.

[37] S. J. Sachdeva, R. Abhishek, and D. A. V.K, "Comparing Machine Learning Techniques for Sentiment Analysis," Ijarcce, vol. 8, no. 4, pp. 67-71, 2019, doi: 10.17148/ijarcce.2019.8410.

[38] M. Khalid, I. Ashraf, A. Mehmood, S. Ullah, M. Ahmad, and G. S. Choi, "GBSVM: Sentiment classification from unstructured reviews using ensemble classifier," Applied Sciences (Switzerland), vol. 10, no. 8, pp. 1-20, 2020, doi: 10.3390/APP10082788.

[39] A. F. Anees, A. Shaikh, A. Shaikh, and S. Shaikh, "Survey Paper on Sentiment Analysis: Techniques and Challenges," EasyChair, pp. 2516-2314, 2020.

[40] L. M. Gómez and M. N. Cáceres, "Applying data mining for sentiment analysis in music," Advances in Intelligent Systems and Computing, vol. 619, pp. 198-205, 2017, doi: 10.1007/978-3-319-61578-3_20.

[41] P. Mehta and S. Pandya, "A review on sentiment analysis methodologies, practices and applications," International Journal of Scientific and Technology Research, vol. 9, no. 2, pp. 601-609, 2020.

[42] A. Sinha, Y. Agrawal, V. Kumar, and C. Kumar, "Survey of Stock Price Prediction Using Sentiment Analysis," no. May, pp. 1140-1143, 2020.

[43] A. Yousif, Z. Niu, J. K. Tarus, and A. Ahmad, "A survey on sentiment analysis of scientific citations," Artificial Intelligence Review, vol. 52, no. 3, pp. 1805-1838, 2019, doi: 10.1007/s10462-017-9597-8.

[44] M. V. Mäntylä, D. Graziotin, and M. Kuutila, "The evolution of sentiment analysis-A review of research topics, venues, and top cited papers," Computer Science Review, vol. 27, no. February, pp. 16-32, 2018, doi: 10.1016/j.cosrev.2017.10.002.

[45] A. Alsaeedi and M. Z. Khan, "A study on sentiment analysis techniques of Twitter data," International Journal of Advanced Computer Science and Applications, vol. 10, no. 2, pp. 361-374, 2019, doi: 10.14569/ijacsa.2019.0100248.

[46] C. Musto, G. Semeraro, and M. Polignano, "A comparison of lexicon-based approaches for sentiment analysis of microblog," CEUR Workshop Proceedings, vol. 1314, pp. 59-68, 2014.

[47] V. A. and S. S. Sonawane, "Sentiment Analysis of Twitter Data: A Survey of Techniques," International Journal of Computer Applications, vol. 139, no. 11, pp. 5-15, 2016, doi: 10.5120/ijca2016908625.

[48] S. W. Reilly and I. Catton, "Utilization of pore-size distributions to predict thermophysical properties and performance of biporous wick evaporators," Journal of Heat Transfer, vol. 136, no. 6, 2014, doi: 10.1115/1.4026624.

[49] S. Goyal, "Review Paper on Sentiment Analysis of Twitter Data Using Text Mining and Hybrid
Classification Approach," Internatio nal Journal of Engineering Development and Research, vol. 5, no. 2, pp. 2321-9939, 2017, [Online]. Available: http://www.ijedr.org/papers/IJEDR1702032.pdf.

[50] H. Xu, B. Liu, L. Shu, and P. S. Yu, "Bert post-training for review reading comprehension and aspect-based sentiment analysis," arXiv, pp. 2324-2335, 2019.

[51] A. P. Patel, A. V. Patel, S. G. Butani, and P. B. Sawant, "Literature Survey on Sentiment Analysis of Twitter Data using Machine Learning Approaches," IJIRST --International Journal for Innovative Research in Science \& Technology, vol. 3, no. 10, pp. 19-21, 2017.

[52] B. Dattu and D. Gore, "A Survey on Sentiment Analysis on Twitter Data Using Different Techniques," International Journal of Computer Science and Information Technologies (IJCSIT), vol. 6, no. 6, pp. 5358-5362, 2015.

[53] N. S, A. Kumar, and A. K K, "Survey on Sentiment Analysis of Stock Market," International Journal of Research-GRANTHAALAYAH, vol. 5, no. 4RACSIT, pp. 69-75, 2017, doi: 10.29121/granthaalayah.v5.i4racsit.2017.3354.

[54] M. Z. Asghar, A. Khan, S. Ahmad, and F. M. Kundi, "A Asghar, M. Z., Khan, A., Ahmad, S., \& Kundi, F. M. (2014). A Review of Feature Extraction in Sentiment Analysis. J. Basic. Appl. Sci. Res, 4(3), 181-186.Review of Feature Extraction in Sentiment Analysis," J. Basic. Appl. Sci. Res, vol. 4, no. 3, pp. 181-186, 2014.

[55] E. Scharifi, A. Danilenko, U. Weidig, and K. Steinhoff, "Influence of plastic deformation gradients at room temperature on precipitation kinetics and mechanical properties of high- strength aluminum alloys," Journal of Engineering Research and Application, vol. 9, no. 1, pp. 24-29, 2019, doi: 10.9790/9622.

[56] V. Uma Ramya and K. Thirupathi Rao, "Sentiment analysis of movie review using machine learning techniques," International Journal of Engineering and Technology(UAE), vol. 7, no. 16, pp. 676-681, 2018, doi: 10.14419/ijet.v7i2.7.10921.

[57] T. C. Asogwa, E. Fidelis, C. Obodoeze, and I. N. Obiokafor, "IJARCCE Wireless Sensor Network (WSN): Applications in Oil \& Gas and Agriculture Industries in Nigeria," International Journal of Advanced Research in Computer and Communication Engineering ISO, vol. 3297, no. 1, pp. 156-159, 2007, doi: 10.17148/IJARCCE.

[58] B. Saberi and S. Saad, "Sentiment analysis or opinion mining: A review," International Journal on Advanced Science, Engineering and Information Technology, vol. 7, no. 5, pp. 1660-1666, 2017, doi: 10.18517/ijaseit.7.5.2137.

[59] S. Salmiah, D. Sudrajat, N. Nasrul, T. Agustin, N. H. Harani, and P. T. Nguyen, "Sentiment analysis for 
amazon products using isolation forest," International Journal of Engineering and Advanced Technology, vol. 8, no. 6 Special issue, pp. 894-897, 2019, doi: 10.35940/ijeat.F1169.0886S19.

[60] Y. Ratawal and D. Tayal, "A Comprehensive study : Sarcasm detection in sentimental analysis," vol. 113, no. October, pp. 218-226, 2018.

[61] N. Nandal, J. Pruthi, and A. Choudhary, "Aspect based sentiment analysis approaches with mining of reviews: A comparative study," International Journal of Recent Technology and Engineering, vol. 7, no. 6, pp. 95-99, 2019.

[62] A. M. Abirami and V. Gayathri, "A survey on sentiment analysis methods and approach," 2016 8th International Conference on Advanced Computing, ICoAC 2016, pp. 72-76, 2017, doi: 10.1109/ICoAC.2017.7951748.

[63] A. H. Shapiro, M. Sudhof, and D. Wilson, "Measuring News Sentiment," Federal Reserve Bank of San Francisco, Working Paper Series, pp. 01-22, 2017, doi: 10.24148/wp2017-01.

[64] A. Kalia, "A Brief Survey Paper on Sentiment Analysis," vol. 3, no. 2, pp. 740-744, 2018.

[65] H. Suzuki, E. Suzuki, W. Chu, J. Terazono, and S. Bhalla, "Exploring kaguya moon mission sensor data by locating geographic entities," Lecture Notes in Computer Science (including subseries Lecture Notes in Artificial Intelligence and Lecture Notes in Bioinformatics), vol. 8883, pp. 169-173, 2014, doi: 10.1007/978-3-319-13820-6.

[66] L. Yue, W. Chen, X. Li, W. Zuo, and M. Yin, "A survey of sentiment analysis in social media," Knowledge and Information Systems, vol. 60, no. 2, pp. 617-663, 2019, doi: 10.1007/s10115-018-1236-4.

[67] P. Baby and K. B, "Sentimental Analysis and Deep Learning: A Survey," International Journal of Scientific Research in Science, Engineering and Technology, pp. 212-220, 2020, doi: 10.32628/ijsrset207135.

[68] M. Gentzkow, B. Kelly, and M. Taddy, "Text as data," Journal of Economic Literature, vol. 57, no. 3, pp. 535-574, 2019, doi: 10.1257/jel.20181020.

[69] L. Zhang, S. Wang, and B. Liu, "Deep learning for sentiment analysis: A survey," arXiv, 2018.

[70] O. Ahlgren, "Research on Sentiment Analysis: The First Decade," IEEE International Conference on Data Mining Workshops, ICDMW, vol. 0, pp. 890-899, 2016, doi: 10.1109/ICDMW.2016.0131.

[71] J. Sadhasivam, R. B. Kalivaradhan, and S. Jayavel, "Survey of various algorithms used in twitter for sentiment analysis," Journal of Critical Reviews, vol. 6, no. 6, pp. 449-454, 2019, doi: 10.31838/jcr.06.06.69.

[72] R. Nimesh, P. Veera Raghava, S. Prince Mary, and B. Bharathi, "A Survey on Opinion Mining and Sentiment Analysis," IOP Conference Series:
Materials Science and Engineering, vol. 590, no. 1, pp. 5075-5078, 2019, doi: 10.1088/1757-899X/590/1/012003.

[73] E. Arts, "a Survey on Lexicon and Machine Learning Based Classification," vol. 6, no. 2, pp. 256-259, 2019.

[74] W. Medhat, A. Hassan, and H. Korashy, "Sentiment analysis algorithms and applications: A survey," Ain Shams Engineering Journal, vol. 5, no. 4, pp. 1093-1113, 2014, doi: 10.1016/j.asej.2014.04.011.

[75] Y. Cui, Q. He, and A. Khani, "Travel Behavior Classification: An Approach with Social Network and Deep Learning," Transportation Research Record, vol. 2672, no. 47, pp. 68-80, 2018, doi: $10.1177 / 0361198118772723$.

[76] A. Kaushik, A. Kaushik, and S. Naithani, "A Study on Sentiment Analysis: Methods and Tools," International Journal of Science and Research (IJSR), vol. 4, no. 12, pp. 287-292, 2015, doi: 10.21275/v4i12.nov151832.

[77] A. Jain and V. Jain, "A Literature Survey on Recommendation System Based on Sentimental Analysis," Advanced Computational Intelligence: An International Journal (ACII), vol. 3, no. 1, pp. 25-36, 2016, doi: 10.5121/acii.2016.3103. 